\title{
Preliminary results of the cohort study of cognitive impairment associated with Parkinson's disease
}

\author{
Lilia Rotaru \\ Laboratory of Functional Neurology, Diomid Gherman Institute of Neurology and Neurosurgery \\ Chisinau, the Republic of Moldova \\ Author's ORCID iD, academic degrees and contribution are available at the end of the article \\ Corresponding author - Lilia Rotaru, e-mail: liliarotaru@yahoo.com \\ Manuscript received July 26, 2021; revised manuscript August 18, 2021; published online September 10, 2021
}

\begin{abstract}
Background: Cognitive impairment (CI) is frequent in Parkinson's disease (PD). CI patients have particular features. These are preliminary data of a cohort study of Moldovan patients with incident Parkinson's disease.

Material and methods: 65 out of 111 consecutive PD patients (mean age $64.87 \pm 7.69$ y.o.; disease duration $50.21 \pm 38.61$ mo.; 48 women (43.2\%), 63 men (56.8\%)) underwent Montreal Cognitive Assessment (MoCA) test. Cognitive status graded as: (1) normal and (2) impaired cognition.

Results: There were similar: ages ( $65.79 \pm 7.13$ vs $62.17 \pm 12.21$ y.o.), onset ages ( $61.44 \pm 7.61$ vs $57.00 \pm 12.95$ years), disease duration ( $49.63 \pm 36.78$ vs $66.00 \pm 26.48)$ months), levodopa (574.58 \pm 129 vs 249.55$)$ and agonists doses (5.19 \pm 3.02 vs $1.05 \pm 0.05)$ and Beck scores (8.13 \pm 6.21 vs $7.4 \pm 3.85)$, in groups. CI was present in $59(90.8 \%)$ patients; more frequent in patients with cardiovascular risk factors $(91.7 \%, \mathrm{p}>0.05)$, symmetrical Parkinsonism (93.2\%, $\mathrm{p}>0.05)$, and in first disease symptom bradykinesia patients (93.8\%, $\mathrm{p}>0.05)$. Upper / Lower Asymmetry Index were lower in CI patients, all lower type patients ( $p>0.05$ ) having CI. MoCA scores correlated with Unified Parkinson's Disease Rating Scale in ON fase (UPDRSon), ( $r=-0.320$, $\mathrm{p}<0.022)$, and red flags number $(\mathrm{r}=-0.590, \mathrm{p}<0.006)$.

Conclusions: Cognitive impairment is more expectable in akinetic, symmetric and lower type Parkinsonism, also in patients with cardiovascular risk factors, with probable PD, and a more motor impairment.

Key words: Parkinson’s disease, cognitive impairment.
\end{abstract}

\section{Cite this article}

Rotaru L. Preliminary results of the cohort study of cognitive impairment associated with Parkinson's disease. Mold Med J. 2021;64(3):40-43. https://doi. org/10.52418/moldovan-med-j.64-3.21.08.

\section{Introduction}

Cognitive impairment (CI) is frequent in Parkinson's disease (PD) even in early stages and over 75\% of PD patients may develop dementia over time [1]. The combination of Lewy pathology and amyloid pathologies is the most robust pathological substrate of PD dementia [2]. Cognitive impairment has been found to be associated with multiple neurotransmitter transmission deficiencies, including dopamine and acetylcholine, indicating a widespread neurotransmitter dysfunction in PD-related dementia [3], but cholinergic deficits due to degeneration of the nucleus basalis of Meynert have been the most involved in this process [4]. Cognitively impaired patients have particular clinical features - older age, postural instability-gait disorder phenotype, psychiatric symptoms (psychosis, hallucinations, and depression), sleep disorders, rapid eye movement (REM) sleep behavior disorder, and diurnal sleepiness); all these factors have been associated with PD cognitive worsening [5]. PD patients with comorbid cardiovascular disorders have an extra risk of developing gait and balance disorders and dementia [5]. A study has shown that PD patients with concurrent cerebral microangiopathy fall more frequently and more often have a disturbed pull test than PD patients with normal CT findings [6]. There is a strong association between the severity of age-related vascular changes as white matter lesions and the severity of gait and motor compromise, probably because white matter lesions may interfere with central processing of sensory-motor signals, leading to impaired gait and postural responses, and resulting in an increased frequency of falls [7].

\section{Material and methods}

These are preliminary data of a cohort study of Moldovan patients with incident Parkinson's disease. Diagnosis of PD was based on widely acknowledged criteria [8]. Structured interview on complaints, medical history and family history of cardiovascular, neurological and psychiatric diseases and drug history was applied; general neurological and medical examinations were conducted. Severity of parkinsonism and disability were assessed by the Modified Unified Parkinson's Disease Rating Scale (MDS-UPDRS) [9]. Cognitive impairment was ascertained by Montreal Cognitive Assessment (MoCA) Scale [10]. Patients were subclassified at baseline into two groups according to MoCA scores: (1) normal cog- 
nition PD patients and (2) cognitively impaired (CI) PD patients. The data analysis was performed via statistical program StatDirect, using descriptive, variation, and correlational analysis. Student's t tests or Mann-Whitney tests were used as appropriate. $\mathrm{P}$ values less than 0.05 were considered statistically significant.

\section{Results and discussion}

The study comprised 111 consecutive PD patients. The mean age in the cohort was $64.87 \pm 7.69$ y.o. Patients had mean disease duration of $50.21 \pm 38.61$ months. Forty-eight patients $(43.2 \%)$ were women and 63 patients $(56.8 \%)$ were men.

Sixty-five out of 111 patients underwent MoCA test. The Montreal Cognitive Assessment has adequate psychometric properties as a screening instrument for the detection of mild cognitive impairment or dementia in Parkinson's disease, in particular for language fluency and visuospatial tests, that are more prevalent in PD [10]. According to their MoCA scores, our PD patients were divided in: (1) normal cognition (control group) and (2) cognitive impaired (CI) patients, with a cut-off of 26/30 [10].

Fifty-nine patients $(90.8 \%)$ of the cognitively tested patients had a MoCA score below 26 and were assigned to the cognitive impaired (CI) group.

Cognitive dysfunction is prevalent in PD. Approximately $10 \%$ of a PD population develop dementia per year and the relative risk for developing dementia in PD compared to non-PD subjects ranges from 1.7 - 5.9 [11]. In Buter T.C. et al. study from a total of 233 PD patients, 140 patients $(60 \%$, $95 \%$ CI $54 \%$ to $66 \%$ ) had developed dementia by the end of the study period, but the cumulative incidence of dementia steadily increased with age and duration of PD to $80 \%-90 \%$ by the age of 90 years; women lived with $\mathrm{PD}$ longer than men and spent more years with dementia [12].

The age of CI patients $(65.79 \pm 7.13$ y.o. $)$ in this study was slightly higher, but similar to those of normal cognition patients $(62.17 \pm 12.21$ y.o., $\mathrm{p}>0.05)$. It is known that aging is a risk factor for mild cognitive impairment [11] and the strongest predictive factor of dementia in PD patients [11]. An argument of association of CI and age comes from a functional RMI study [13] which proves age-related reduction of hub function in the medial prefrontal cortex. The results of a study [11] provide, that having mild CI, was associated with: older age at assessment, older age at disease onset, male gender, depression, more severe motor symptoms, and advanced disease stage.

In the present study PD onset ages (61.44 \pm 7.61 vs 57.00 \pm 12.95 years, $\mathrm{p}>0.05)$ and disease duration $(49.63 \pm 36.78$ vs $66.00 \pm 26.48$ months, $\mathrm{p}>0.05$ ) in CI patients were similar to normal cognition patients.

Levodopa equivalent daily doses $(574.58 \pm 129$ vs 249.55 mg, $\mathrm{p}>0.05)$ and agonists doses $(5.19 \pm 3.02$ vs $1.05 \pm 0.05$ $\mathrm{mg}, \mathrm{p}>0.05$ ) were slightly higher, but without statistic difference between study and control group. Levodopa (L-dopa) is the gold standard treatment for Parkinson's disease and the use of L-dopa in patients with Parkinsonism with dementia does not adversely affect cognitive function. Molloy S.A. et al. also established in their study, that the mean daily dose of L-dopa was larger in patients with PD dementia (672 (451) $\mathrm{mg}$ ) than in those with Parkinson's disease (407 (197) mg, $\mathrm{p}=0.02$ ) with normal cognition [14]. The explanation may be more motor impairment associated with $\mathrm{CI}$ in PD.

The most common clinically relevant neuropsychiatric symptoms in PD are apathy (38.9\%), depression (34.5\%), and anxiety (23.8\%) [15]. Depressive symptoms in Parkinson's disease correlate with impaired global and specific cognitive performance [16]. When screened for depression, patients with CI of this study, had insignificantly higher Beck scores than patients without CI $(8.13 \pm 6.21$ vs $7.4 \pm 3.85, \mathrm{p}>0.05)$. As usual, depressive symptoms correlate with global cognitive performance, but naming, verbal memory, and language are the most susceptible affected cognitive domains [16].

The present research revealed that CI was more frequent in patients with cardiovascular risk factors (according to QRISK3 list [17]). So, 91.7\% of patients having one or more vascular risk factors, had emphasized CI on MoCA test, vs $80.0 \%$ of those not reporting vascular risk factors ( $p>0.05)$. According to recent data, a third of all dementia cases may be preventable, due to cardiovascular risk factors, and trials targeting primarily cardiovascular risk factors show improved cognitive function in people at risk [18]. In both sexes, 10-year absolute risk of all-cause dementia increases with increasing age, number of apolipoprotein $\mathrm{E}$ (APOE) $\varepsilon 4$ alleles, number of genome-wide association studies (GWAS) risk alleles, and presence of cardiovascular risk factors [18].

Forty-one of our patients (93.2\%) with symmetrical Parkinsonism (according to their right/left asymmetry index) had CI (vs $18 \mathrm{p}(85.7 \%, \mathrm{p}>0.05)$. In patients reporting bradykinesia as the first disease symptom, 30 patients (93.8\%) developed CI, in contrast to 23 patients $(85.2 \%)$, reporting tremor as the first disease symptom ( $p>0.05$ ).

Alves G. et al. found that in nearly all dementia cases, dementia was preceded by PIGD (postural instability gait disorder) or a kinetic dominant or by the transfer from tremor-dominant (TD) to PIGD type PD. The odds ratio for dementia was 56.7 (95\% CI: 4.0-808.4; P = 0.003) for patients changing from TD or indeterminate subtype to PIGD subtype, and 80.0 (95\% CI: 4.6-1400.1; P = 0.003) for patients with persistent PIGD subtype (compared to patients with persistent TD or indeterminate subtype). Patients with TD subtype at baseline become demented only when they developed PIGD subtype; dementia did not occur among patients with persistent TD subtype of Parkinsonism [19].

Upper / Lower Asymmetry Indexes ( $0.60 \pm 0.37$ vs $2.4 \pm$ 0.97, $\mathrm{p}>0.05$ ) were lower in CI patients indicating a relative symmetric involvement of limbs.

According to the " $a$-Synuclein Origin and Connectome Model of Parkinson's Disease Motor Asymmetry", "bodyfirst" patients at onset (initial origin of $\alpha$-Synuclein pathology in peripheral nervous system spreading bilateral 
to brain) already have a larger, more symmetric burden of a-Synuclein pathology, which in turn promotes symmetric faster disease progression and accelerated cognitive decline [20].

In the current study, patients with predominant involvement of lower limbs, according to their superior/inferior asymmetry index, were assigned as lower type Parkinsonism patients. All lower type Parkinsonism patients of this study (15 - 100\%) developed CI, in contrast to control (18 $85.6 \%), \mathrm{p}>0.05$.

Estimates of the frequency of lower type Parkinsonism, indicating a vascular origin vary greatly worldwide: from $3 \%$ to $6 \%$ of all Parkinsonism cases [21]. Attention and memory impairment were found in $73.5 \%$ of patients with vascular Parkinsonism after mean disease duration of $4.4 \pm 3.4$ years in Colosimo C. et al. study [22].

MoCA and Unified Parkinson's Disease Rating Scale in ON fase (UPDRSon) scores in this study were inversely correlated - patients with more severe motor impairment had lower cognitive scores, hence more expressed cognitive impairment $(\mathrm{r}=-0.320, \mathrm{p}<0.022)$.

According to a recent study, female gender and education supported cognitive function in PD, while age and depression scores were inversely correlated with overall cognition; and UPDRS had little influence on cognition; the conclusion of that study being that education, aging, comorbidity, and gender may impact cognition more than overall disease severity in PD [23]. However, another study on 2327 PD patients with a disease duration of over 20 years, found that demented PD patients exhibited more severe motor features compared to non-demented PD patients [24]. Memory impairment was most common $(13.3 \%$; 11.6-15.3), followed by visuospatial $(11.0 \% ; 9.4-13.0)$ and attention/executive ability impairment (10.1\%; 8.6-11.9); (11.3\% 9.7-13.1) were classified as nonamnestic single-domain MCI, $8.9 \%$ (7.0-9.9) as amnestic single-domain, 4.8\% (3.8-6.1) as amnestic multiple-domain, and $1.3 \%(0.9-2.1)$ as nonamnestic multiple-domain. MoCA scores are lower in PD, particularly for language fluency and visuospatial tests [10].

There were recorded the presence and number of red flags, according to MDS clinical diagnostic criteria for Parkinson's disease [25]. The number of red flags also correlated with MoCA scores. number $(r=-0.590, p<0.006)$, indicating that a lower degree of certainty of Parkinson's disease diagnosis is associated with a more pronounced cognitive deficit.

\section{Conclusions}

According to the presented data, patients with Parkinson's disease and cognitive impairment, displayed no difference regarding ages, onset ages and disease duration compared to the controls. Cognitive impairment was more prevalent in akinetic PD phenotype, as well as in symmetric and lower type Parkinsonism. Patients having cardiovascular risk factors more frequently had cognitive impairment as well; and cognitive dysfunction was associated with more pronounced motor impairment.

\section{References}

1. Santangelo G, Vitale C, Picillo M, et al. Mild Cognitive Impairment in newly diagnosed Parkinson's disease: a longitudinal prospective study. Parkinsonism Relat Disord. 2015;21(10):1219-1226. doi: 10.1016/j. parkreldis.2015.08.024.

2. Smith C, Malek N, Grosset K, et al. Neuropathology of dementia in patients with Parkinson's disease: a systematic review of autopsy studies. J Neurol Neurosurg Psychiatry. 2019;90(11):1234-1243. doi: 10.1136/ jnnp-2019-321111.

3. Silbert LC, Kaye J. Neuroimaging and cognition in Parkinson's disease dementia. Brain Pathol. 2010;20(3):646-653. doi: 10.1111/j.17503639.2009.00368.x.

4. Mattila PM, Röyttä M, Lönnberg P, et al. Choline acetyltransferase activity and striatal dopamine receptors in Parkinson's disease in relation to cognitive impairment. Acta Neuropathol. 2001;102:160-166. doi: 10.1007/s004010100372.

5. Painous C, Marti MJ. Cognitive impairment in Parkinson's disease : what we know so far. Res Reviews Parkinsonism. 2020;10:7-17. https://doi. org/10.2147/JPRLS.S263041.

6. Ebersbach G, Sojer M, Müller J, et al. Gleichgewichtsstörungen bei idiopathischer Parkinson-Erkrankung: Der Einfluss zerebrovaskulärer Komorbidität [Dysequilibrium in Parkinson's disease: influence of cerebrovascular comorbidity]. Nervenarzt. 2002;73:162-165. doi: 10.1007/ s00115-001-1234-7. German.

7. Baezner H, Blahak C, Poggesi A, et al. Association of gait and balance disorders with age-related white matter changes: the LADIS study. Neurology. 2008;70(12):935-942. doi: 10.1212/01.wnl.0000305959.46197.e6.

8. Postuma RB, Poewe W, Litvan I, et al. Validation of the MDS clinical diagnostic criteria for Parkinson's disease. Mov Disord. 2018;33(10):16011608. doi: $10.1002 / \mathrm{mds} .27362$

9. Goetz CG, Tilley BC, Shaftman SR, et al. Movement Disorder SocietySponsored Revision of the Unified Parkinson's Disease Rating Scale (MDS-UPDRS): scale presentation and clinimetric testing results. Mov Disord. 2008;23:2129-2170. doi: 10.1002/mds.22340.

10. Kasten M, Bruggemann N, Schmidt A, et al. Validity of the MoCA and MMSE in the detection of MCI and dementia in Parkinson's disease. Neurology. 2010;75(5):478-479. doi: 10.1212/WNL.0b013e3181e7948a.

11. Aarsland D, Kurz MW. The epidemiology of dementia associated with Parkinson's disease. J Neurol Sci. 2010;289(1-2):18-22. doi: 10.1016/j. jns.2009.08.034.

12. Buter TC, van den Hout A, Matthews FE, et al. Dementia and survival in Parkinson's disease: a 12-year population study. Neurology. 2008;70(13):1017-1022. doi: 10.1212/01.wnl.0000306632.43729.24.

13. Nagano-Saito A, Bellec P, Hanganu A, et al. Why is aging a risk factor for cognitive impairment in Parkinson's disease? - A resting state fMRI study. Front Neurol. 2019;10:267. doi: 10.3389/fneur.2019.00267.

14. Molloy SA, Rowan EN, O'Brien JT, et al. Effect of levodopa on cognitive function in Parkinson's disease with and without dementia and dementia with Lewy bodies. J Neurol Neurosurg Psychiatry. 2006;77(12):13231328. doi: 10.1136/jnnp.2006.098079.

15. Hommel ALAJ, Meinders MJ, Lorenzl S, et al. The prevalence and determinants of neuropsychiatric symptoms in late-stage Parkinsonism. Mov Disord Clin Pract. 2020;7(5):531-542. doi: 10.1002/mdc3.12968.

16. Fernandez HH, See RH, Gary MF, et al. Depressive symptoms in Parkinson's disease correlate with impaired global and specific cognitive performance. J Geriatr Psychiatry Neurol. 2009;22(4):223-227. doi: 10.1177/0891988709335792.

17. Hippisley-Cox J, Coupland C, Brindle P. Development and validation of QRISK3 risk prediction algorithms to estimate future risk of cardiovascular disease: prospective cohort study. BMJ. 2017;357:1-21. doi: 10.1136/bmj.j2099.

18. Juul Rasmussen I, Rasmussen KL, Nordestgaard BG, et al. Impact of cardiovascular risk factors and genetics on 10-year absolute risk of dementia: risk charts for targeted prevention. Eur Heart J. 2020;41(41):4024-4033. doi: 10.1093/eurheartj/ehaa695.

19. Alves G, Larsen JP, Emre M, et al. Changes in motor subtype and risk for incident dementia in Parkinson's disease. Mov Disord. 2006;21(8):1123 1130. doi: $10.1002 / \mathrm{mds} .20897$. 
20. Borghammer P. The $\alpha$-Synuclein Origin and Connectome Model (SOC Model) of Parkinson's disease: explaining motor asymmetry, non-motor phenotypes, and cognitive decline. J Parkinsons Dis. 2021;11(2):455-474. doi: 10.3233/JPD-202481.

21. Vale TC, Barbosa MT, Caramelli P, et al. Vascular Parkinsonism and cognitive impairment: literature review, Brazilian studies and case vignettes. Dement Neuropsychol. 2012;6(3):137-144. doi: 10.1590/ S1980-57642012DN06030005.

22. Colosimo C, Morgante L, Antonini A, et al. Non-motor symptoms in atypical and secondary Parkinsonism: the PRIAMO study. J Neurol. 2010;257(1):5-14. doi: 10.1007/s00415-009-5255-7.
23. Lin SJ, Baumeister TR, Garg S, et al. Cognitive profiles and hub vulnerability in Parkinson's disease. Front Neurol. 2018;9:1-13. doi: 10.3389/ fneur.2018.00482.

24. Szeto JYY, Walton CC, Rizos A, et al. Dementia in long-term Parkinson's disease patients: a multicentre retrospective study. NPJ Park Dis. 2020;6:2. doi: 10.1038/s41531-019-0106-4.

25. Postuma RB, Berg D, Stern M, et al. MDS clinical diagnostic criteria for Parkinson's disease. Mov Disord. 2015;30(12):1591-1601. doi: 10.1002/ mds.26424.

\section{Author's ORCID iD and academic degrees}

Lilia Rotaru, MD, PhD, Associate Professor - https://orcid.org/0000-0002-5340-5234

\section{Author's contribution}

AC conceptualized the idea, conducted literature review, wrote the manuscript, revised and finalized the text.

\section{Funding}

This study was supported by Diomid Gherman Institute of Neurology and Neurosurgery. The trial was the author's initiative. The author is independent and takes responsibility for the integrity of the data and accuracy of the data analysis.

\section{Ethics approval and consent to participate}

The research project was approved by the Research Ethics Committee of Diomid Gherman Institute of Neurology and Neurosurgery (protocol No 1, 27.02.2020).

\section{Conflict of Interests}

No competing interests were disclosed. 